\title{
A short time interval between radiotherapy and hyperthermia reduces in-field recurrence and mortality in women with advanced cervical cancer
}

Caspar M. van Leeuwen ${ }^{1 *}$, Arlene L. Oei ${ }^{1,2^{*}}$, Kenneth W. T. K. Chin ${ }^{1}$, Johannes Crezee ${ }^{1}$, Arjan Bel', Anneke M. Westermann ${ }^{3}$, Marrije R. Buist ${ }^{4}$, Nicolaas A. P. Franken ${ }^{1,2}$, Lukas J. A. Stalpers ${ }^{1}$ and H. Petra Kok ${ }^{1}$

\begin{abstract}
Background: Combined radiotherapy and hyperthermia is a well-established alternative to chemoradiotherapy for advanced stage cervical cancer patients with a contraindication for chemotherapy. Pre-clinical evidence suggests that the radiosensitizing effect of hyperthermia decreases substantially for time intervals between radiotherapy and hyperthermia as short as $1-2 \mathrm{~h}$, but clinical evidence is limited. The purpose of this study is to determine the effect of the time interval between external beam radiotherapy (EBRT) and same-day hyperthermia on in-field recurrence rate, overall survival and late toxicity in women with advanced stage cervical cancer.

Methods: Patients with advanced stage cervical cancer who underwent a full-course of curative daily EBRT and (4-5) weekly hyperthermia sessions between 1999 and 2014 were included for retrospective analysis. The mean time interval between EBRT fractions and same-day hyperthermia was calculated for each patient; the median thereof was used to divide the cohort in a 'short' and 'long' time-interval group. Kaplan-Meier analysis and stepwise Cox regression were used to compare the in-field recurrence and overall survival. Finally, high-grade $(\geq 3)$ late toxicity was compared across time-interval groups. DNA repair suppression is an important hyperthermia mechanism, DNA damage repair kinetics were therefore studied in patient biopsies to support clinical findings.
\end{abstract}

Results: Included were 58 patients. The 3-year in field recurrence rate was 18\% and 53\% in the short ( $\leq 79.2 \mathrm{~min}$ ) and long (>79.2 min) time-interval group, respectively $(p=0.021)$; the 5 -year overall survival was $52 \%$ and $17 \%$ respectively $(p=0.015)$. Differences between time-interval groups remained significant for both in-field recurrence ( $H R=7.7, p=0.007)$ and overall survival $(H R=2.3, p=0.012)$ in multivariable Cox regression. No difference in toxicity was observed $(p=1.00)$, with only 6 and 5 events in the short and long group, respectively. The majority of DNA damage was repaired within $2 \mathrm{~h}$, potentially explaining a reduced effectiveness of hyperthermia for long time intervals.

Conclusions: A short time interval between EBRT and hyperthermia is associated with a lower risk of in-field recurrence and a better overall survival. There was no evidence for difference in late toxicity.

Keywords: Radiotherapy, Hyperthermia, Time interval, Clinical outcome, Cervical cancer

\footnotetext{
*Correspondence: c.m.vanleeuwen@amc.uva.nl; a.l.oei@amc.uva.nl

'Department of Radiation Oncology, Academic Medical Center, University of

Amsterdam, Meibergdreef 9, 1105 AZ Amsterdam, The Netherlands

Full list of author information is available at the end of the article
} 


\section{Background}

Cervical cancer is the fourth most common cancer in women worldwide, with 528,000 new cases and 266,000 deaths in 2012 [1]. Standard treatment for locally advanced cervical cancer is radiotherapy combined with weekly cisplatin-based chemotherapy [2]. Thermoradiotherapy, i.e. radiotherapy combined with hyperthermia, is a well-established alternative for patients with a contraindication for chemotherapy and provides similar overall survival [3-5].

Clinical thermoradiotherapy generally consists of fractionated daily external beam radiotherapy (EBRT) and, during the same period, weekly hyperthermia. In hyperthermia, the tumor is heated to a temperature of $40-43{ }^{\circ} \mathrm{C}$ for one hour. The rationale for adding hyperthermia to radiotherapy is that hyperthermia suppresses DNA double strand break (DSB) repair, the most lethal type of DNA damage caused by radiation treatment $[6,7]$. Additionally, hyperthermia also sensitizes radioresistent (hypoxic) tumors by increasing oxygen delivery $[8,9]$. This radiosensitizing effect increases the efficacy of the radiation treatment [10-13]. An EBRT fraction and hyperthermia session are usually given sequentially rather than simultaneously, since preclinical studies suggest that this results in the best therapeutic ratio $[10,14]$.

In clinical practice, the time interval between EBRT and hyperthermia treatment typically varies from $0.5-4 \mathrm{~h}$ for various reasons [5], such as availability of the treatment machines. Pre-clinical data suggest that longer time intervals between radiotherapy and hyperthermia reduce the radiosensitizing effect of thermoradiotherapy [14, 15], but clinical evidence is scarce. Only two studies investigated the effect of time interval, and only for a small and heterogeneous series of superficial tumors $[16,17]$.

Aim of this study is to determine the effect of the time interval between EBRT and hyperthermia treatments on in-field recurrence, overall survival and late toxicity in a retrospective cohort of cervical cancer patients. Furthermore, by examining the effect of time interval between EBRT and hyperthermia on the prevalence of DSBs in patient biopsies we study a potential mechanism supporting the clinically observed relationship.

\section{Methods}

\section{Patient population}

Included were patients treated at the Academic Medical Center for cervical cancer (ICD-9: 180, ICD-10: C53) with curative thermoradiotherapy, between January 1999 and January 2014. Excluded were patients who received concurrent chemotherapy and patients who received less than four out of the intended five hyperthermia sessions. Patients who received EBRT at other institutes were also excluded, because variation between institutes (e.g. different treatment guidelines, radiation schedules and techniques) would have introduced too many potential confounding factors.

All patients had a histologically confirmed cervical carcinoma, and were staged by FIGO clinical staging, including investigation under general anesthesia with cystoscopy, and lymph node staging by imaging (CT, MRI and/or PET). Patients with bulky lymph nodes $(>2 \mathrm{~cm})$ received a lymph node debulking first. Patients were referred for primary radiotherapy with hyperthermia for locally inoperable tumors (large FIGO IIB tumors, IIIA, IIIB and IVA) and for lymph node positive patients with FIGO IB and IIA. Since 2001, chemoradiotherapy became the standard treatment, and thermoradiotherapy was reserved for patients with a medical contraindication for cisplatin-based chemotherapy (i.e. hydronephrosis, renal insufficiency, poor performance).

In-field recurrence, overall survival and late toxicity data were extracted from patient files. Subsequently, overall survival for patients who were Dutch citizens was updated using the Dutch civil registry. Late toxicities, occurring or persisting at least 6 months after completion of thermoradiotherapy, were scored according to CTCAE v4.0. Only high-grade $(\geq 3)$ toxicities were analysed, since retrospective analysis of low-grade toxicity is less reliable.

\section{Treatment}

Treatment consisted of daily EBRT (23 x 2 Gy or $28 \times 1.8 \mathrm{~Gy}$ ) and five (occasionally four) weekly locoregional hyperthermia treatments. At the end of the treatment period, a pulsed dose rate brachytherapy boost was given (24 Gy). Initially, EBRT was delivered using 3D conformal techniques, with a transition to IMRT in 2011. Hyperthermia was delivered during the period of EBRT treatment, approximately $1 \mathrm{~h}$ after the corresponding EBRT fraction. Hyperthermia was delivered by the AMC4 phased array system, a $70 \mathrm{MHz}$ radiofrequency heating system designed for deep-seated tumors [18]. A water bolus is attached to each antenna, to couple the electric field into the tissue and to cool the skin. An intravaginal E-field probe at the tumor location was used to determine the phase settings that yield optimal target heating. Temperatures were monitored during treatment using intracavitary 14-sensor thermocouple probes (spacing $0.5 \mathrm{~cm}$, accuracy $\pm 0.01{ }^{\circ} \mathrm{C}$, ELLA, Czech Republic), placed in the vagina, bladder and rectum. Sensors located at positions indicative for the tumor were labelled as tumor in the monitoring software. Temperatures were measured every $30 \mathrm{~s}$ after a $5 \mathrm{~s}$ power off to avoid electromagnetic disturbance [19]. A steady state duration of $60 \mathrm{~min}$ was aimed for. Start of the steady state was defined as the moment when (after an initial warm-up period) one of the temperature sensors in the target region reached $41{ }^{\circ} \mathrm{C}$, or 
30 min after the start of the warm-up period if a temperature of $41{ }^{\circ} \mathrm{C}$ was not reached within that time.

\section{Statistical analysis}

Since multiple hyperthermia treatments are delivered, treatment of a patient is not characterized by a single time interval between EBRT and hyperthermia. Thus, for each patient, the mean time interval $\left(t_{\text {int,mean }}\right)$ between their hyperthermia treatments and corresponding EBRT fractions was calculated. The median of $t_{\text {int,mean was }}$ then used to split the population into a 'short' and a 'long' time-interval group.

The following patient and treatment characteristics were described for each time-interval group. Pretreatment variables: age, histology, FIGO stage, lymph node status, smoking status. Hyperthermia parameters: tumor temperature $\left(\mathrm{T}_{90 \text {,mean }}\right)$, the steady state duration (HT duration mean $)$, warm-up time, and number of hyperthermia treatments. $\mathrm{T}_{90}$ represents the temperature reached in at least $90 \%$ of the temperature measurement locations that are indicative for the tumor. $\mathrm{T}_{90 \text {,mean }}$ is the mean $\mathrm{T}_{90}$ over each patient's hyperthermia treatment series. Warm-up time was defined as the time between the start of power on to the start of the steady state. Differences between both time-interval groups in terms of these patient and treatment characteristics were tested for using Fisher's exact test, the Chi-square test, the independent samples $t$-test and the Mann-Whitney $U$ test depending on the type of data.

In-field recurrence rate and overall survival were calculated by the Kaplan-Meier method, and groups were compared by the log-rank test. Time to event (in-field recurrence or death) and censoring were calculated from the date of diagnosis. Multivariable analysis of in-field recurrence and overall survival was done by (backwards) stepwise Cox regression, including time-interval group,

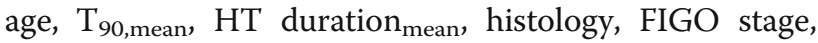
lymph node status, number of hyperthermia treatments and smoking status as factors. A Fisher's exact test was used to test for differences in the incidence of highgrade toxicity between time-interval groups. All analyses were performed using SPSS version 23, all tests were two-sided and $p<0.05$ was considered significant. Accuracy of statistical estimates is reported using $95 \%$ confidence intervals.

\section{Patient biopsies}

An effect of time interval on clinical outcome could be related to the amount of unrepaired DNA DSBs present at the time the hyperthermia is given, since DSB repair suppression is an important mechanism for the radiosensitizing effect of hyperthermia. To investigate this, experiments were performed on 12 cervical carcinoma biopsies. Biopsies were obtained from patients diagnosed in 2015 with advanced stage cervical cancer and eligible for thermoradiotherapy. Biopsies were divided in two parts: one half was treated ex vivo with radiotherapy (4 Gy), the other was left untreated (control). Six samples were fixated at approximately 15 min and six samples at approximately $2 \mathrm{~h}$ after radiotherapy. After treatment, biopsies were submerged in paraformaldehyde, to be used for paraffin coupes. Before antigen retrieval, they were deparaffinized and rehydrated. Afterwards a heat-induced antigen retrieval at $\mathrm{pH} 9.0$ for 20 min was performed, followed by a 30 min cooling period. Next, a 15 min PO block including $\mathrm{H}_{2} \mathrm{O}_{2}$ was performed. Then coupes were incubated overnight at $4{ }^{\circ} \mathrm{C}$ with $\gamma$-H2AX mAb (Millipore, Merck). Next, tissue was embedded in Alexa Fluor 488 (Invitrogen Life Technologies), after washing with PBS. DAPI was used to stain the nuclei blue before covering tissue with a drop of ProLong Gold anti-fade reagent (Invitrogen Life Technologies) and a coverslip.

\section{Results}

Fifty-eight patients were included. The median time interval was $79.2 \mathrm{~min}$, defining the short and long timeinterval groups as $t_{\text {int,mean }} \leq 79.2 \mathrm{~min}(33.8-79.2 \mathrm{~min})$ and $t_{\text {int,mean }}>79.2 \mathrm{~min}(80.0-125.2 \mathrm{~min})$ respectively. Out of all clinical and treatment characteristics, only warm-up time and the time interval itself were significantly different across time-interval groups (Table 1). Median follow-up for censored patients was 18 months (range, 2-130 months) for in-field recurrence and 37 months (range, 3-195 months) for overall survival.

The in-field recurrence rate and overall survival were significantly better in the short time interval group (Fig. 1). The 3-year in-field recurrence rate was $18 \%$ (0$35 \%)$ in the short time-interval group and 53\% (18-82\%) in the long time-interval group. The 5 -year overall survival was $52 \%(35-77 \%)$ in the short time-interval group and $17 \%(7-41 \%)$ in the long time-interval group; median overall survival was 61 months (38-83 months) and 19 months (13-26 months) respectively.

The last iteration of the stepwise Cox regression for in-field recurrence included three significant factors for a favorable outcome: a short time interval, advanced age and long warm-up time (Table 2). In the overall survival analysis, the last iteration included three prognostically favorable factors: a short time interval (significant), high $\mathrm{T}_{90 \text {,mean }}$ (significant) and negative lymph node status (trend, not significant).

Six high-grade late toxicities were observed in the short time-interval group, compared to five high-grade toxicities in the long time-interval group (Table $3, p=1.00$ ).

The $\gamma$-H2AX staining of patient biopsies showed a substantial increase in DSBs for the six samples fixated 15 min after irradiation, compared to control (Fig. 2a). For the six samples fixated at two hours, the number of 
Table 1 Characteristics of the included patients, stratified by the mean time interval between radiotherapy and hyperthermia

\begin{tabular}{|c|c|c|c|c|c|c|}
\hline & \multicolumn{2}{|c|}{ Short group $(n=30)$} & \multicolumn{2}{|c|}{ Long group $(n=28)$} & Statistical test & $p$ \\
\hline & \multicolumn{2}{|c|}{ Median (range) } & \multicolumn{2}{|c|}{ Median (range) } & & \\
\hline$t_{\text {int,mean }}[\mathrm{min}]$ & \multicolumn{2}{|c|}{$65.8(33.8-79.2)$} & \multicolumn{2}{|c|}{$91.7(80.0-125.2)$} & Mann-Whitney $U$ test & $<0.001$ \\
\hline Age $[y]$ & \multicolumn{2}{|c|}{$67.5(33-90)$} & \multicolumn{2}{|c|}{$65.0(29-85)$} & Mann-Whitney $U$ test & 0.45 \\
\hline $\mathrm{T}_{90, \text { mean }}\left[{ }^{\circ} \mathrm{C}\right]$ & \multicolumn{2}{|c|}{$40.0(38.6-41.9)$} & \multicolumn{2}{|c|}{$40.3(38.2-41.1)$} & T-test & 0.71 \\
\hline HT duration ${ }_{\text {mean }}[\mathrm{min}]$ & \multicolumn{2}{|c|}{$60.0(52.6-63.8)$} & \multicolumn{2}{|c|}{$60.8(34.5-64.6)$} & Mann-Whitney $U$ test & 0.16 \\
\hline \multirow[t]{2}{*}{ Warm-up time [min] } & \multicolumn{2}{|c|}{$5.2(0.75-17.8)$} & \multicolumn{2}{|c|}{$8.5(2.75-26.6)$} & Mann-Whitney $U$ test & 0.001 \\
\hline & N & $\%$ & N & $\%$ & & \\
\hline Histology & & & & & Fisher's exact test & 1.00 \\
\hline Squamous cell carcinoma & 27 & 90 & 26 & 93 & & \\
\hline Adenocarcinoma & 3 & 10 & 2 & 7 & & \\
\hline Figo stage & & & & & Chi-square test & 0.40 \\
\hline $\mathrm{IB}$ & 3 & 10 & 4 & 14 & & \\
\hline$\| \mathrm{A}$ & 1 & 3 & 0 & 0 & & \\
\hline$\| B$ & 6 & 20 & 8 & 29 & & \\
\hline$\| I I A$ & 2 & 7 & 5 & 18 & & \\
\hline$\| I \mid B$ & 14 & 47 & 10 & 36 & & \\
\hline IVA & 4 & 13 & 1 & 4 & & \\
\hline Lymph node status & & & & & Fisher's exact test & 1.00 \\
\hline Negative & 15 & 50 & 14 & 50 & & \\
\hline Positive & 15 & 50 & 14 & 50 & & \\
\hline Number of hyperthermia treatments & & & & & Fisher's exact test & 0.22 \\
\hline 4 & 5 & 17 & 9 & 32 & & \\
\hline 5 & 25 & 83 & 19 & 68 & & \\
\hline Smoking & & & & & Fisher's exact test & 1.00 \\
\hline Yes & 7 & 23 & 7 & 25 & & \\
\hline No & 23 & 77 & 21 & 75 & & \\
\hline
\end{tabular}

DSBs was similar to that of untreated samples (Fig. 2b). Patients included in this retrospective study had time intervals between EBRT and hyperthermia ranging from 30 min to two hours. Thus, patients with short time intervals received hyperthermia when substantial DNA damage was still present, while patients with long time
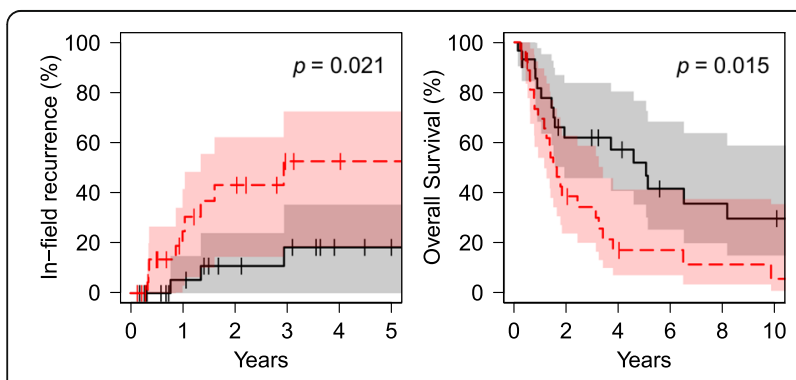

Fig 1 Kaplan-Meier survival analysis for in-field recurrence (left) and overall survival (right) for the short time-interval group (black) and the long time-interval group (red). Shaded area represents the 95\% confidence interval intervals received hyperthermia when the majority of DNA damage was already repaired.

\section{Discussion}

This is the first clinical study to demonstrate the effect of time interval on treatment outcome in patients with advanced stage cervical cancer. The results of both univariable and multivariable analyses indicate that a short

Table 2 Last iterations of the backwards stepwise Cox regression for in-field recurrence and overall survival

\begin{tabular}{llll}
\hline In-field recurrence & Factor & $\mathrm{HR}(95 \% \mathrm{Cl})$ & $p$ \\
& Time-interval group & $7.7(1.8-33.8)$ & 0.007 \\
& Age & $0.96(0.92-1.00)$ & 0.048 \\
& Warm-up time & $0.85(0.72-1.00)$ & 0.047 \\
& & & \\
Overall survival & Time-interval group & $2.3(1.2-4.5)$ & 0.012 \\
& To0,mean & $0.57(0.39-0.87)$ & 0.009 \\
& Nodal status & $1.8(0.91-3.4)$ & 0.093 \\
\hline
\end{tabular}


Table 3 Number and type of late toxicities, stratified by timeinterval group

\begin{tabular}{lll}
\hline CTC-score & Short group & Long group \\
\hline$<3$ & Unspecified (16) & Unspecified (17) \\
3 & $\begin{array}{l}\text { Radiation cystitis (1), } \\
\text { Pelvic fracture (1), }\end{array}$ & Local radiation ulcer (1) \\
& $\begin{array}{l}\text { rectovaginal fistula (1), } \\
\text { local radiation ulcer (1) }\end{array}$ \\
& $\begin{array}{l}\text { Secondary in-field } \\
\text { malignancy (1), local }\end{array}$ & $\begin{array}{l}\text { Radiation enteritis (1), } \\
\text { complex/multiple (1), } \\
\end{array}$ \\
& radiation ulcer (1) & vesicovaginal fistula (1) \\
5 & - & Gastrointestinal perforation (1) \\
Insufficient & 8 & 6 \\
follow-up & & \\
\hline
\end{tabular}

time interval between EBRT and hyperthermia treatments results in a lower in-field recurrence rate and better overall survival. Although the confidence intervals were substantial due to the limited size of the patient group, the estimated effect size was large enough to yield a significant result. At the same time, the results provide no evidence for an effect of time interval on high-grade late toxicity; a possible relationship may however have been obscured by the low number of toxicity events.

In addition to time-interval group, a significant correlation between age and in-field recurrence rate was identified in the multivariable analysis, with older patients having less recurrences (Table 2). This effect may be explained by our patient policy. While elderly women were often denied chemotherapy due to their generally frail condition, younger patients usually received thermoradiotherapy instead of chemoradiotherapy because of hydronephrosis. This results in a selection of young patients with relatively large local tumors and/or extensive lymph node metastasis. Warm-up time was also identified as a significant factor, where patients with longer warm-up time did better. After warm-up, a fixed duration of steady state $(60 \mathrm{~min})$ is aimed for. Thus, patients with longer warm-up time will have a longer total heating time, and have therefore received a higher thermal dose. Comparing the patients in our study with the shortest and longest warmup times (approximately 1$25 \mathrm{~min}$ ), the difference in CEM43 was about 10-15\%. The observed significance of warm-up time can therefore be understood in view of the well-established correlation between thermal dose and clinical outcome [20-25]. However, the estimated hazard ratio (0.85 $\mathrm{min}^{-}$ ${ }^{1}$ ) is uncertain (i.e. has a large $95 \%$ confidence interval) and appears to be relatively low considering the modest difference in CEM43. For overall survival, a high $\mathrm{T}_{90, \text { mean }}$ was identified as a significantly favorable factor, also supporting a thermal dose effect relationship.

Biological studies in the late 70's already suggested that the time interval between irradiation and hyperthermia affects outcome $[14,15]$. These in vitro and in vivo experiments have shown that the radiosensitizing effect of hyperthermia in tumor tissue decays substantially in the

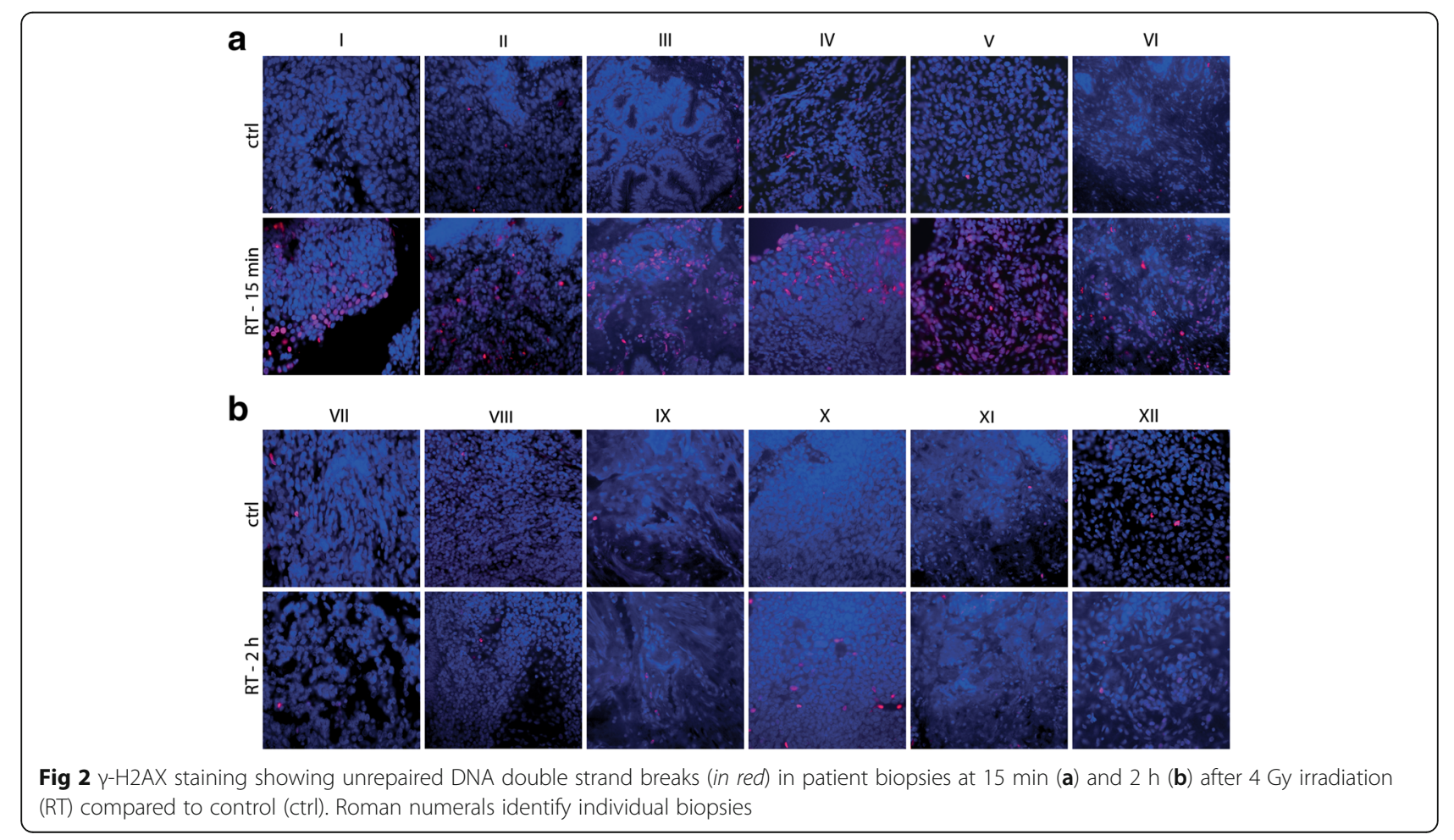


first $2 \mathrm{~h}$, in particular when EBRT is given before hyperthermia (Fig. 2 in Li et al [15] and Fig. 5 in Overgaard [14]). These pre-clinical experiments were performed at higher temperatures $\left(42.5-43{ }^{\circ} \mathrm{C}\right)$ than what is generally achieved in the clinic, and the contribution of the various mechanisms of hyperthermia (e.g. DNA repair inhibition, reoxygenation and direct cytotoxicity) may be different at lower temperatures [26]. Nonetheless, these data corroborate the difference in local control observed in this study. Additionally, Overgaard's data suggest that the radiosensitizing effect decays even more rapidly in normal tissue, regardless of the order in which radiotherapy and hyperthermia are applied. If a substantial part of the radiosensitizing effect in normal tissue has already disappeared for a time interval of one hour, this could explain why no difference in late toxicity was observed between the short time-interval group (median $65.8 \mathrm{~min}$ ) and the long time-interval group (median $91.7 \mathrm{~min})$.

Prior to this study, clinical evidence on the effect of time interval was limited to the results of two small studies, which both investigated superficial recurrent and metastatic tumors of mixed primary origin. Lindholm et al compared time intervals of $0.5-1.5 \mathrm{~h}$ to $3-$ $4 \mathrm{~h}$ and saw no significant difference between both groups in terms of either tumor response or skin toxicity [16]. However, the short time-interval group in this study only included 15 tumors and time-interval groups were not comparable with respect to the numbers and mode of hyperthermia treatment. Arcangeli et al described three trials involving hyperthermia [17]. The second trial compared hyperthermia immediately after radiotherapy, with delayed hyperthermia ( $4 \mathrm{~h}$ between treatments). Reported local tumor control at 6 months was $5 / 7$ for immediate hyperthermia compared to $4 / 7$ for delayed hyperthermia. Moist desquamation occurred in $64 \%$ and $46 \%$ of the cases for immediate and delayed hyperthermia respectively. However, definite conclusions could not be drawn because of the small numbers. Additionally, the results of both studies may not be applicable to modern hyperthermia, because of substantial improvements in hyperthermia treatment techniques resulting in better treatment quality.

In a more recent study, a more homogenous group of superficial tumors (all recurrent breast cancers) was studied [27]. Hyperthermia was applied after radiation and one factor that was investigated was whether patients received EBRT and hyperthermia in the same or in different institutes. For patients treated within a single institute, local control was worse (not significant) and late toxicity was increased (significant). However, treatment outcomes generally vary between institutes due to e.g. differences in patient selection and treatment. Thus, the actual effect of time interval is very difficult to determine from these results, since any relation between the factor 'institute' and treatment outcome cannot be exclusively attributed to an effect of time interval.

Multiple mechanisms have been suggested for the radiosensitizing effect of hyperthermia [26]. In our institute, hyperthermia is delivered after radiation, and the ability of heat to interfere with DNA damage repair is an important mechanism for radiosensitization for this treatment sequence $[6,7]$. This mechanism can only be effective if unrepaired DNA damage is still present. Radiobiological studies on clinical data have shown that the exponential repair time constant for radiation damage is roughly $1.5 \mathrm{~h}$ [28-31]. A similar repair time is suggested by the results of our experiments on patient biopsies. While a substantial amount of DNA DSBs was observed $15 \mathrm{~min}$ after irradiation (Fig. 2a), almost all DNA damage was repaired after two hours (Fig. 2b). As time intervals in the long time-interval group ranged up to $2 \mathrm{~h}$, our biopsy data could explain why hyperthermia is less effective in this group: since much of the radiation damage has already been repaired when hyperthermia is given, the efficacy of the repair-blocking mechanism is substantially reduced. In contrast, a substantial amount of unrepaired damage is still present at the time of hyperthermia treatment in the short time-interval group, thus the repair-blocking mechanism is effective. It is important to note that when radiation is delivered after heat, increased perfusion and corresponding reoxygenation may become more important mechanisms for radiosensitization than DNA repair inhibition [32]. Thus, for this order of treatments, the dynamics of radiosensitization may be different from what was observed in this study and additional research is needed to determine the effect of time interval for hyperthermia applied before radiation.

The Dutch Deep Hyperthermia Trial (DDHT), which compared radiotherapy to thermoradiotherapy in advanced stage cervical cancer, reported a 3-year overall survival of $27 \%$ for the radiotherapy group and $51 \%$ for the thermoradiotherapy group [33]. In our study, the 3year overall survival for all 58 patients was 48\% (36$64 \%$ ), almost similar as in the DDHT. However, 3-year overall survival was considerably lower in the long timeinterval group at $34 \%(20-59 \%)$ compared to $62 \%$ (46$84 \%)$ in the short time-interval group. Comparison of the two series could lead to two conclusions. First, the overall survival in the long time-interval group in this study was close to that of the radiotherapy-alone arm in the DDHT trial, suggesting that long time-interval patients had very little benefit from the hyperthermia treatment. Second, if a short time interval can be ensured for all patients, an additional improvement of approximately $10 \%$ in overall survival of the results in the DDHT may be attained. 
Our findings may have important consequences for treatment policy of women with inoperable cervical cancer. Although underpowered, results from a trial comparing standard chemoradiotherapy with thermoradiotherapy in women with inoperable cervical cancer suggest that chemoradiotherapy and thermoradiotherapy are equally effective, with approximately $60 \%$ long term event free survival in both arms [5]. However, time interval between EBRT and hyperthermia in this trial ranged from $1-4 \mathrm{~h}$, which may have resulted in sub-optimal results for the thermoradiotherapy group. Thus, thermoradiotherapy could potentially be even more effective than standard chemoradiotherapy, provided a short time interval between EBRT and hyperthermia is ensured. This hypothesis would need confirmation through a clinical trial, and would require EBRT and hyperthermia to be delivered with a short time interval.

Recently, a systematic review showed the potential value of thermochemoradiotherapy in the treatment of locally advanced cervical cancer, but concluded that further confirmation through prospective randomized trials is needed [34]. This raises the question whether the effect of time interval between radiotherapy and hyperthermia is equally important in thermochemoradiotherapy, in which case imposing strict limits to the time interval should be considered in designing such a trial. Hyperthermia and cisplatin primarily inhibit different DNA repair pathways (homologues recombination and non-homologous endjoining, respectively) $[7,35,36]$. Although blocking additional repair pathways may cause activation or a shift to other pathways (potentially causing a change in dynamics), it seems unlikely that the dynamics of the interaction between hyperthermia and radiotherapy would be changed dramatically. While no definitive proof is available, limiting the time interval seems a reasonable approach when aiming at an optimal synergistic action of the three modalities in a thermochemoradiotherapy trial.

The importance of a short time interval has implications for clinical practice. In recent years an increasing number of patients received hyperthermia at our institute, but radiation treatment elsewhere (5 out of $11 \mathrm{pa}$ tients in 2014). This is more convenient for patients, since the hyperthermia center (generally a longer commute than the nearest radiotherapy center) then only needs to be visited once a week. However, considering the substantial reduction in efficacy of the hyperthermia, delivering both treatments in separate institutes should be strongly discouraged. A solution would be for patients to receive EBRT on the day of hyperthermia within the same institute, while all other EBRT treatments are delivered in a center closer to the patients residence. However, this requires radiotherapy treatment plans to be designed for both institutes and will yield additional workload. Even when both treatments are given within a single institute, long time intervals should be avoided, and this is now standard practice for locoregional hyperthermia at our institute. While the optimal time interval cannot be established based on the current data and an increase in normal toxicity may be expected for very short time intervals based on pre-clinical data, a time interval of $1 \mathrm{~h}$ appears to be a reasonable tradeoff between feasibility, efficacy and safety $[14,15]$.

\section{Conclusion}

A short time interval between EBRT and hyperthermia is associated with a lower risk of in-field recurrence and a better overall survival. Efficacy is reduced for longer time intervals, likely because of the reduced amount of unrepaired DNA damage present at the time of hyperthermia treatment. There was no evidence for a difference in long-term toxicity, however, the low number of events in both arms means that statistical power is limited. Limiting the time interval between EBRT and hyperthermia to approximately one hour is recommended.

\section{Abbreviations}

DDHT: Dutch deep hyperthermia trial; DSB: double strand break; EBRT: external beam radiotherapy

\section{Acknowledgements}

Not applicable

\section{Funding}

This work was financially supported by the Dutch Cancer Society KWF, grant UVA 2012-5540

\section{Availability of data and materials}

The datasets generated and/or analysed during the current study are available from the corresponding author on reasonable request.

\section{Author's contributions}

CML carried out the writing of the manuscript. CML, KWC, LS and ALO contributed to the aggregation and analysis of the retrospective data. MRB provided the patient biopsies. ALO carried out experiments on patient biopsies. JC assisted in the design of the manuscript. HPK provided general supervision and assisted in the design of the manuscript. All authors participated in the drafting and revising of the manuscript. All authors read and approved the final manuscript.

\section{Competing interests}

The authors declare that they have no competing interests.

\section{Consent for publication}

Not applicable

Ethics approval and consent to participate

This study was approved by the AMC's Medical Ethics Committee (AMC/MEC 03/137). Informed consent was obtained from all patients for the collection of biopsies; the Medical Ethics Committee waived the requirement for informed consent for the retrospective part of this study.

\section{Publisher's Note}

Springer Nature remains neutral with regard to jurisdictional claims in published maps and institutional affiliations.

\section{Author details}

${ }^{1}$ Department of Radiation Oncology, Academic Medical Center, University of Amsterdam, Meibergdreef 9, 1105 AZ Amsterdam, The Netherlands. 
${ }^{2}$ Laboratory for Experimental Oncology and Radiobiology (LEXOR)/Center for Experimental Molecular Medicine, Academic Medical Center, University of Amsterdam, Meibergdreef 9, 1105 AZ Amsterdam, The Netherlands. ${ }^{3}$ Department of Medical Oncology, Academic Medical Center, University of Amsterdam, Meibergdreef 9, 1105 AZ Amsterdam, The Netherlands. ${ }^{4}$ Department of Obstetrics and Gynecology, Center for Gynecologic Oncology Amsterdam, Academic Medical Center, Meibergdreef 9, 1105 AZ Amsterdam, The Netherlands.

Received: 30 September 2016 Accepted: 25 April 2017 Published online: 27 April 2017

\section{References}

1. Ferlay J, Soerjomataram I, Dikshit R, Eser S, Mathers C, Rebelo M, et al. Cancer incidence and mortality worldwide: Sources, methods and major patterns in GLOBOCAN 2012. Int J Cancer. 2015;136:E359-86. doi:10.1002/ijc.29210.

2. Chemoradiotherapy for Cervical Cancer Meta-analysis Collaboration (CCCMAC). Reducing uncertainties about the effects of chemoradiotherapy for cervical cancer: individual patient data meta-analysis. Cochrane Database Syst Rev. 2010;26:CD008285. doi:10.1002/14651858.CD008285.

3. Lutgens L, van der Zee J, Pijls-Johannesma M, De Haas-Kock DF, Buijsen J, Mastrigt GA van, et al. Combined use of hyperthermia and radiation therapy for treating locally advanced cervical carcinoma. Cochrane Database Syst Rev 2010:CD006377. doi:10.1002/14651858.CD006377.pub2.

4. Eifel PJ, Winter K, Morris M, Levenback C, Grigsby PW, Cooper J, et al. Pelvic irradiation with concurrent chemotherapy versus pelvic and para-aortic irradiation for high-risk cervical cancer: an update of radiation therapy oncology group trial (RTOG) 90-01. J Clin Oncol. 2004;22:872-80. doi:10. 1200/JCO.2004.07.197.

5. Lutgens LCHW, Koper PCM, Jobsen JJ, van der Steen-Banasik EM, Creutzberg $\mathrm{CL}$, van den Berg HA, et al. Radiation therapy combined with hyperthermia versus cisplatin for locally advanced cervical cancer: Results of the randomized RADCHOC trial. Radiother Oncol. 2016;120:378-82. doi:10. 1016/j.radonc.2016.02.010.

6. Oei AL, Vriend LEM, Crezee J, Franken NAP, Krawczyk PM. Effects of hyperthermia on DNA repair pathways: one treatment to inhibit them all. Radiat Oncol. 2015;10:165. doi:10.1186/s13014-015-0462-0.

7. Krawczyk PM, Eppink B, Essers J, Stap J, Rodermond H, Odijk H, et al. Mild hyperthermia inhibits homologous recombination, induces BRCA2 degradation, and sensitizes cancer cells to poly (ADP-ribose) polymerase-1 inhibition. Proc Natl Acad Sci USA. 2011;108:9851-6. doi:10.1073/pnas.1101053108.

8. Vujaskovic Z, Song CW. Physiological mechanisms underlying heat-induced radiosensitization. Int J Hyperthermia. 2004;20:163-74. doi:10.1080/ 02656730310001619514.

9. Sun $X$, Xing L, Ling CC, Li GC. The effect of mild temperature hyperthermia on tumour hypoxia and blood perfusion: relevance for radiotherapy, vascular targeting and imaging. Int J Hyperthermia. 2010;26:224-31. doi:10. 3109/02656730903479855.

10. Horsman MR, Overgaard J. Hyperthermia: a potent enhancer of radiotherapy. Clin Oncol. 2007;19:418-26. doi:10.1016/j.clon.2007.03.015.

11. Wust P, Hildebrandt B, Sreenivasa G, Rau B, Gellermann J, Riess H, et al. Hyperthermia in combined treatment of cancer. Lancet Oncol. 2002;3:487-97. doi:10.1016/S1470-2045(02)00818-5.

12. Crezee J, van Leeuwen $C M$, Oei AL, van Heerden $L E$, Bel A, Stalpers $\sqcup A$, et al. Biological modelling of the radiation dose escalation effect of regional hyperthermia in cervical cancer. Radiat Oncol. 2016;11:14. doi:10.1186/s13014-016-0592-z.

13. Cihoric N, Tsikkinis A, van Rhoon G, Crezee H, Aebersold DM, Bodis S, et al. Hyperthermia-related clinical trials on cancer treatment within the ClinicalTrials.gov registry. Int J Hyperthermia. 2015;31:609-14. doi:10.3109/ 02656736.2015.1040471.

14. Overgaard J. Simultaneous and sequential hyperthermia and radiation treatment of an experimental tumor and its surrounding normal tissue in vivo. Int J Radiat Oncol Biol Phys. 1980;6:1507-17. doi:10.1016/03603016(80)90008-5.

15. Li GC, Kal HB. Effect of hyperthermia on the radiation response of two mammalian cell lines. Eur J Cancer. 1977:13:65-9. doi:10.1016/00142964(77)90231-6

16. Lindholm CE, Kjellen E, Nilsson P, Hertzman S. Microwave-induced hyperthermia and radiotherapy in human superficial tumours: clinical results with a comparative study of combined treatment versus radiotherapy alone. Int J Hyperthermia. 1987;3:393-411. doi:10.3109/02656738709140410.
17. Arcangeli G, Cividalli A, Nervi C, Creton G, Lovisolo G, Mauro F. Tumor control and therapeutic gain with different schedules of combined radiotherapy and local external hyperthermia in human cancer. Int J Radiat Oncol Biol Phys. 1983;9:1125-34. doi:10.1016/0360-3016(83)90170-0.

18. van Dijk JD, Schneider C, van Os R, Blank LE, Gonzalez DG. Results of deep body hyperthermia with large waveguide radiators. Adv Exp Med Biol. 1990; 267:315-9. doi:10.1007/978-1-4684-5766-7_31.

19. De Leeuw AA, Crezee J, Lagendijk JJ. Temperature and SAR measurements in deep-body hyperthermia with thermocouple thermometry. Int J Hyperthermia. 1993;9:685-97. doi:10.3109/02656739309032056.

20. Franckena M, Fatehi D, de Bruijne M, Canters RAM, van Norden Y, Mens JW, et al. Hyperthermia dose-effect relationship in 420 patients with cervical cancer treated with combined radiotherapy and hyperthermia. Eur I Cancer. 2009:45:1969-78. doi:10.1016/j.ejca.2009.03.009.

21. Wust P, Rau B, Gellerman J, Pegios W, Löffel J, Riess H, et al. Radiochemotherapy and hyperthermia in the treatment of rectal cancer. Recent Results Cancer Res. 1998;146:175-91. doi:10.1007/978-3-642-71967-7_16.

22. Sherar M, Liu FF, Pintilie M, Levin W, Hunt J, Hill R, et al. Relationship between thermal dose and outcome in thermoradiotherapy treatments for superficial recurrences of breast cancer: data from a phase III trial. Int J Radiat Oncol Biol Phys. 1997;39:371-80. doi:10.1016/50360-3016(97)00333-7.

23. Overgaard J, Gonzalez Gonzalez D, Hulshof MC, Arcangeli G, Dahl O, Mella O, et al. Hyperthermia as an adjuvant to radiation therapy of recurrent or metastatic malignant melanoma. A multicentre randomized trial by the European Society for Hyperthermic Oncology. Int J Hyperthermia. 1996;12:3-20. doi:10.1080/ 02656730903091986

24. Myerson RJ, Perez CA, Emami B, Straube W, Kuske RR, Leybovich L, et al. Tumor control in long-term survivors following superficial hyperthermia. Int J Radiat Oncol Biol Phys. 1990;18:1123-9. doi:10.1016/0360-3016(90)90448-S.

25. Oleson JR, Samulski TV, Leopold KA, Clegg ST, Dewhirst MW, Dodge RK, et al. Sensitivity of hyperthermia trial outcomes to temperature and time: implications for thermal goals of treatment. Int I Radiat Oncol Biol Phys. 1993;25:289-97. doi:10.1016/0360-3016(93)90351-U.

26. Crezee $H$, van Leeuwen $C M$, Oei AL, Stalpers $\perp A$, Bel A, Franken NA, et al. Thermoradiotherapy planning: Integration in routine clinical practice. Int J Hyperthermia. 2016;32:41-9. doi:10.3109/02656736.2015.1110757.

27. Linthorst M, van Geel AN, Baaijens M, Ameziane A, Ghidey W, van Rhoon GC, et al. Re-irradiation and hyperthermia after surgery for recurrent breast cancer. Radiother Oncol. 2013;109:188-93. doi:10.1016/j.radonc.2013.05.010.

28. Kal HB, Van Gellekom MPR. How low is the alpha/beta ratio for prostate cancer? Int J Radiat Oncol Biol Phys. 2003;57:1116-21. doi:10.1016/503603016(03)01455-X.

29. Fowler J, Chappell R, Ritter M. Is alpha/beta for prostate tumors really low? Int J Radiat Oncol Biol Phys. 2001;50:1021-31. doi:10.1016/S03603016(01)01607-8.

30. Roberts SA, Hendry JH, Swindell R, Wilkinson JM, Hunter RD. Compensation for changes in dose-rate in radical low-dose-rate brachytherapy: a radiobiological analysis of a randomised clinical trial. Radiother Oncol. 2004; 70:63-74. doi:10.1016/j.radonc.2003.11.010.

31. Joiner MC, Bentzen SM. Fractionation: the linear-quadratic approach. In: Joiner MC, van der Kogel AJ, editors. Basic Clin Radiobiol. 4th ed. London: Hodder Arnold; 2009. p. 102-19.

32. Dewhirst MW, Lee C-T, Ashcraft KA. The future of biology in driving the field of hyperthermia. Int J Hyperthermia. 2016;32:4-13. doi:10.3109/02656736. 2015.1091093.

33. van der Zee J, González D, van Rhoon GC, van Dijk JD, van Putten WL, Hart AA. Comparison of radiotherapy alone with radiotherapy plus hyperthermia in locally advanced pelvic tumours: a prospective, randomised, multicentre trial. Lancet. 2000;355:1119-25. doi:10.1016/S0140-6736(00)02059-6.

34. Datta NR, Rogers S, Klingbiel D, Gómez S, Puric E, Bodis S. Hyperthermia and radiotherapy with or without chemotherapy in locally advanced cervical cancer: a systematic review with conventional and network meta-analyses. Int J Hyperthermia. 2016;32:809-21. doi:10.1080/02656736.2016.1195924.

35. Myint WK, Ng C, Raaphorst GP. Examining the non-homologous repair process following cisplatin and radiation treatments. Int J Radiat Biol. 2002; 78:417-24. doi:10.1080/09553000110113047.

36. Diggle CP, Bentley J, Knowles MA, Kiltie AE. Inhibition of double-strand break non-homologous end-joining by cisplatin adducts in human cell extracts. Nucleic Acids Res. 2005;33:2531-9. doi:10.1093/nar/gki528. 